\title{
Single spin detection by qubit SWAP to a molecular nanomagnet
}

\author{
M. Feng ${ }^{1,2} *$ and J. Twamley $1 \dagger$ \\ ${ }^{1}$ Department of Mathematical Physics, National University of Ireland, Maynooth, Co. Kildare, Ireland \\ 2 Wuhan Institute of Physics and Mathematics, Chinese Academy of Sciences, Wuhan, 430071, China
}

(Dated: September 13, 2018)

\begin{abstract}
Spin state detection is a key but very challenging step for any spin-based solid-state quantum computing technology. In fullerene based quantum computer technologies, we here propose to detect the single spin inside a fullerene by transferring the quantum information from the endohedral spin to the ground states of a molecular nanomagnet $\mathrm{Fe}_{8}$, with large spin $\mathrm{S}=10$. We show how to perform the required SWAP operation and how to read out the information through state-of-the-art techniques such as micro-SQUID.
\end{abstract}

PACS numbers: 03.67.Lx, 73.21.-b

\section{INTRODUCTION}

Among the variety of promising technologies to carry out quantum information processing, spin-based solidstate qubit technologies have great appeal through the potential use of large-scale fabrication techniques to scale up a quantum computer design [1]. Although there have been a number of proposals for carrying out universal quantum gates in this respect, the read out of the information from individual spin-based qubits - the very last and necessary step in any quantum processor routine - is still a major difficulty.

The main idea of current theoretical proposals for single spin detection is to convert spin-state encoded quantum information into charge state encodings. Since the energy of a multi-electron system is spin-dependent, due to the Pauli exclusion principle, one can engineer a current whose spin polarization depends on spin-qubit state and thus provides a measurement of the electronic spin state 2, 3]. Experimentally, Scanning- Tunneling- Microscope electron spin resonance (ESR) has reached the single spin level in the case of iron impurities in Silicon [4]. However the precise mechanism responsible for this effect is not yet well understood. Magnetic Resonance Force Microscopy may also become a useful technique for single electron spin detection where the cantilever oscillation is resonantly driven by a single spin 5 . However this technique will require further development before this technique reaches single spin sensitivity although significant advances have been recently demonstrated [ $[\underline{\text { ] }}$. Recently, the movement of individual electrons has been evidenced by a $C_{60}$-based single electron transistor through electromechanical coupling 7]. It can be shown that this arrangement can be used as a single spin detector in the presence of an external magnetic field 8 ].

Although our focus here is to use a Micro-SQUID (Superconducting Quantum Interference Device - a device capable of distinguishing large spin difference $(\Delta m=30)$

\footnotetext{
*Electronic address: mfeng@thphys.may.ie

$\dagger$ Electronic address: Jason.Twamley@MAY.IE
}

[9]), we argue that to obtain sufficient sensitivity it will be useful to combine this device with methods which could convert the small spin- $1 / 2$ qubit signal into a system with a much larger spin. What we want to detect is the $m_{s}$ component of the electronic spin state of the dopant atom inside a fullerene $C_{60}$. This doped fullerene or endohedral fullerene is the primary host for quantum information in a number of fullerene based quantum computer designs which include the endohedral molecules $N @ C_{60}$ or $P @ C_{60}[10,11,12]$. In these types of designs, the quantum information is encoded in the nuclear spin of the endohedral atom. The execution of quantum gates utilises the hyperfine interaction and magnetic dipolar coupling via the application of standard nuclear magnetic resonance (NMR) and ESR pulse sequences. It has been clearly demonstrated in [12] that fullerene - based quantum computing meets the requirement for a quantum computer, except for the lack of an effective readout technique. Since the nuclear spin is less sensitive to the external environment, our detection of a single spin state would be carried out on the electronic spin state after the nuclear spin states have been swapped to the electronic spin states.

Since the problem we consider is only related to the readout of quantum information from a single electron spin state, we will neglect nuclear spins in this paper. There are three valence electrons in the dopant atom $N$ or $P$, whose ground state in the presence of magnetic field is $|\uparrow \uparrow \uparrow\rangle$. Because other excited states are of much higher energy, the only spin state suitable for encoding qubits is $|\uparrow \uparrow \uparrow\rangle$, which can be considered as a single spin$3 / 2$ state with quartet components $\left|m_{s}= \pm 3 / 2\right\rangle$ and $\left|m_{s}= \pm 1 / 2\right\rangle$ in a magnetic field. It has been shown in 12 that universal quantum information processing can be carried out independently with inner qubits $| \pm 1 / 2\rangle$ or outer qubits $| \pm 3 / 2\rangle$. Therefore what we want to detect is whether the inner qubit is in $|1 / 2\rangle$ or $|-1 / 2\rangle$ or whether the outer qubit is in $|3 / 2\rangle$ or $|-3 / 2\rangle$. 


\section{COUPLING SYSTEM OF A FULLERENE AND A $\mathrm{Fe}_{8}$}

Since the electrons of the dopant atoms of $C_{60}$ are always trapped in the cage made of 60 carbon atoms, the qubit detection mechanisms suggested in 2, 3], cannot be applied here as these endohedral electrons cannot be liberated from the cage without destroying the delicate qubit information encoded in their $m_{s}$ spin states. So the key step of our scheme is to swap the encapsulated spin state to an outside auxiliary spin state which is easily detectable. We consider the following Hamiltonian describes the endohedral electronic system coupled to a nearby auxiliary large-spin system,

$$
H=H_{1}+H_{2}+H_{I}
$$

where $H_{1}=-g_{1} \mu_{B} B_{z} S_{1 z}$ denotes the spin (to be detected), inside the fullerene with $g_{1}=2, \mu_{B}$ being the Bohr magneton, $\mathrm{B}_{z}$ being the magnetic field strength along z-axis, and $S_{1 z}$ the Pauli matrix for $S=3 / 2 . H_{2}$ is related to the auxiliary system with large spin and $H_{I}$ is the coupling between the two systems in a magnetic field.

We employ a molecular nanomagnet made of $\mathrm{Fe}_{8}[13$, which is small but has a relatively high magnetic moment, as the auxiliary system. It has already been shown that one can experimentally prepare a single $\mathrm{Fe}_{8}$ crystal in the ground states $| \pm 10\rangle$ at very low temperatures $(<360 \mathrm{mK})$ 14]. It has also been shown that one can observe the quantum tunneling of magnetization in a single $\mathrm{Fe}_{8}$ crystal, which will prove useful for our SWAP operation. The lowest energy levels of $\mathrm{Fe}_{8}$ can be described as a spin-10 Hamiltonian [14, 15]

$$
H_{2}=-D S_{2 z}^{2}+H^{\text {tran }}-g_{2} \mu_{B} B_{z} S_{2 z},
$$

where $D \approx 0.275 \mathrm{~K}$ is the axial anisotropy constant and $g_{2} \approx g_{1}$. In what follows, we define $\omega=g \mu_{B} B_{z}$ in units of $\hbar=1$, where $g=g_{1} \approx g_{2}$. $S_{2 z}$ is the Pauli matrix for $S=10 . H^{\text {tran }}$ is the transverse anisotropy due to the applied magnetic field in $\mathrm{x}-\mathrm{y}$ plane, which yields the tunnel splitting [16]. The exact form of $H^{\text {tran }}$ is not important in our discussion. Moreover, the term concerning $S_{z}^{4}$ whose coefficient is much smaller than D is omitted in Eq. (2). We suppose that the detected endohedral spin is coupled to the auxiliary spin of the molecular magnet by magnetic dipolar interaction, which is generally described as $H_{I}=J_{0}(A+B+C+E+F+G)$ where $A=$ $\left(1-3 \cos ^{2} \theta\right) S_{1 z} \otimes S_{2 z}, B=-(1 / 4)\left(1-3 \cos ^{2} \theta\right)\left(S_{1+} \otimes\right.$ $\left.S_{2-}+S_{1-} \otimes S_{2+}\right), C=-(3 / 2) \sin \theta \cos \theta e^{-i \phi}\left(S_{1 z} \otimes S_{2+}+\right.$ $\left.S_{1+} \otimes S_{2 z}\right), E=C^{*}, F=-(3 / 4) \sin ^{2} \theta e^{-i 2 \phi} S_{1+} \otimes S_{2+}$, and $G=-(3 / 4) \sin ^{2} \theta e^{i 2 \phi} S_{1-} \otimes S_{2-}$ with $\theta$ and $\phi$ the usual polar and azimuth angles, respectively.

Eq. (11) can be exactly solved by numerical computation. For simplicity, however, we will investigate the system under consideration following the assumptions below: (i) The transverse anisotropy terms will be neglected in the following deduction as done in [17], because it is not essential to our conclusion. (We will go beyond this assumption when discussing the quantum tunneling of magnetization.) (ii) Only the Ising-type term $A$, related to $S_{1 z} \otimes S_{2 z}$, will be retained in $\mathrm{H}_{I}$, which is valid in the weak coupling limit [12]. As we will show below, in our implementation, the detuning is much larger than $\mathrm{J}_{0}$, so we can safely omit the terms in $\mathrm{H}_{I}$ other than $A$. As a result, the Hamiltonian under consideration can be simply rewritten as

$$
H_{c}=-\omega\left(S_{1 z}+S_{2 z}\right)-D S_{2 z}^{2}+J S_{1 z} \otimes S_{2 z},
$$

where $J=J_{0}\left(1-3 \cos ^{2} \theta\right)$ and we assume $J=0.0175 \mathrm{~K}$ [18]. Since all the terms in Eq. (3) are diagonal, it is easy to obtain the eigenstates as well as the corresponding eigenenergies, as shown in Table \

\section{SWAP IMPLEMENTATION}

Our scheme consists of two essential steps: the first step is to swap the detected endohedral electronic spin states inside the fullerene to the ground states $| \pm 10\rangle$ of $\mathrm{Fe}_{8}$. Then as the second step, we measure the states $| \pm 10\rangle$ from $\mathrm{Fe}_{8}$. Since a SWAP consists of three controlled-NOT (CNOT) operations, we will investigate in this section how to carry out CNOT within our model. To this end, we will make use of the quantum tunneling of magnetization in a single $\mathrm{Fe}_{8}$ molecule.

1. Implementation of $C N O T_{21}$. Table \shows the existence of degenerate transitions in the subspace regarding $H_{1}$. These transitions are heavily dependent on the coupling (or neighboring) spin states in $H_{2}$. Based on this characteristic, the irradiation of a ESR pulse with a resonant frequency on the first spin yields, in the interaction representation, an effective Hamiltonian $(\hbar=1)$ for the subsystem of $H_{1}$,

$$
\tilde{H}=\Omega S_{1 x},
$$

where $\Omega$ is the Rabi frequency,

$$
S_{1 x}=\frac{1}{2}\left(\begin{array}{cccc}
0 & \sqrt{3} & 0 & 0 \\
\sqrt{3} & 0 & 2 & 0 \\
0 & 2 & 0 & \sqrt{3} \\
0 & 0 & \sqrt{3} & 0
\end{array}\right),
$$

and the resonant frequency is one of the degenerate transition frequencies labeled in Table【 For a $\pi$ pulse irradiation of ESR, i.e. $\Omega t=\pi, H_{I}$ yields the operator

$$
\hat{P}=i\left(\begin{array}{cccc}
0 & 0 & 0 & 1 \\
0 & 0 & 1 & 0 \\
0 & 1 & 0 & 0 \\
1 & 0 & 0 & 0
\end{array}\right)
$$

which works independently in the subspace spanned by $| \pm 3 / 2\rangle$ or the one spanned by $| \pm 1 / 2\rangle$.

Therefore, with the operator $\hat{P}$, we can flip states $| \pm 3 / 2\rangle$ or $| \pm 1 / 2\rangle$ of a single qubit with an ESR pulse 
whose frequency is determined by the neighboring spin state in $\mathrm{H}_{2}$. That is actually a $\mathrm{CNOT}_{21}$ operation. Theoretically, the fidelity of this selective pulse method depends on more detailed characteristics of the physically coupled systems. The implementation time is determined by the Rabi frequency $\Omega$.

2. Implementation of $\mathrm{CNOT}_{12}$. Due to the term $S_{2 z}^{2}$, the levels in $\mathrm{Fe}_{8}$ are not equidistant and so $\mathrm{CNOT}_{12}$ cannot be carried out by the above method with selective ESR pulses. Let us simply consider the case of very low temperature, in which only the ground states $| \pm 10\rangle$ of $H_{2}$ are populated. By considering $| \pm 10\rangle$ coupled with the possible qubit states of $\mathrm{H}_{1}$, we show the magnetic field dependence of the energy in Fig. 1, where the displayed crossing points correspond to the doublet degenerate cases. However, if we include the neglected term $H^{\text {tran }}$ in our calculation, all of these crossing points would turn into avoided crossings due to the tunneling splitting. So by sweeping the magnetic field $\mathrm{B}_{z}$ through these (avoided) crossing points, we should have tunneling between different magnetization states. For example, with the magnetic field $\mathrm{B}_{z}$ swept through $0.019 \mathrm{~T}$ (from $0.019_{-} \mathrm{T}$ to $0.019_{+} \mathrm{T}$ ), we have the magnetization tunneling from $|3 / 2,-10\rangle$ to $|3 / 2,10\rangle$. Note that in Fig. 1 the (avoided) crossing points of different kinds of lines mean the places where the magnetization tunneling occurs with very small probability due to the second or higher-order process, e.g. $|1 / 2,10\rangle \rightarrow|3 / 2,-10\rangle$. Moreover, since the eigenenergies associated with $|n, m\rangle, \quad n=$ $\pm 1 / 2, \pm 3 / 2$ and $m= \pm 9, \pm 8, \ldots$ are much higher, and thus are physically hard to reach in the low temperature case, we omit them in Fig. 1.

The fidelity of our scheme is affected by the following: (1) We must have precise knowledge of the dipolar coupling strength $J$, as this determines the quality of the CNOT gates we perform. The $J$-value can be obtained experimentally by interrogative ESR pulses. (2) We must have very small linewidth selective ESR pulses. Since the wavelength of the ESR pulse is much larger than the distance between $\mathrm{Fe}_{8}$ and C60, for avoiding exciting $\mathrm{Fe}_{8}$ when we perform $\mathrm{CNOT}_{21}$, it is required that the linewidths of ESR pulses be narrower than $J$, the minimal difference between degenerate transition frequencies. (3) We must accurately control the tunneling time in the performance of $\mathrm{CNOT}_{12}$. This time depends on the sweep rate of the magnetic field and the tunnel splitting. We prefer a fast tunneling to make the implementation time of SWAP shorter than the decoherence time. In this case, we need a large tunnel splitting at the avoided crossing points.

\section{DETECTION OF STATES $| \pm 10\rangle$}

Due to the very high sensitivity (which reaches $10^{-16}$ electromagnetic units 19]), and from the full spectrum analysis we have done for nanomagnets [20], the microSQUID can hopefully be used to measure the spin states of single $\mathrm{Fe}_{8}$ crystals directly by standard spectroscopy with pulsed ESR [15].

\section{DISCUSSION AND CONCLUSION}

Although the experimental value of the coupling $J$ is not yet known, we expect $J$ to be suitably large for our purpose in implementing the CNOT gates. First, as mentioned above, the prerequisite of a perfect $\mathrm{CNOT}_{21} \mathrm{im}$ plementation is that the linewidth of the ESR pulse is smaller than $J$. So the larger the coupling $J$, the less strict the requirement for the linewidth of the ESR pulse. Secondly, a larger coupling $J$ is advantageous to achieve more accurate implementations of the magnetization tunneling. In terms of our numerical calculation, if $J$ is very close to zero, all the (avoided) crossing points would be nearly overlapping. It implies that our scheme would not work because we could not distinguish different qubit spin states from the tunneling signal. On the other hand, $J$ cannot be too large. To keep the weak coupling limit in Eq. (3) valid, $|J|$ should be much smaller than $\min \{|\omega|$, D $\}$. In our case with $J=0.0175 \mathrm{~K}$ corresponding to 350 $\mathrm{MHz}, \mathrm{ESR}$ pulses with much narrower linewidths have already been achieved experimentally [21].For the magnetization tunneling $|3 / 2,-10\rangle \leftrightarrow|3 / 2,10\rangle$ occurring at $B_{z}=0.019 \mathrm{~T},|J| \sim|\omega| / 2 \ll D$. Nevertheless, as long as the dipolar angle $\theta$ in $H_{I}$ is close to $\pi / 2$, terms $C$ and $E$ in $\mathrm{H}_{I}$ would be nearly zero, and other terms (except $A$ ) could be neglected in the weak limit and so Eq. (3) still holds.

An essential point for implementing our scheme is that the SWAP time should be shorter than the decoherence time of the system. For $\mathrm{Fe}_{8}$, the $\mathrm{T}_{1}$ times for the $| \pm 10\rangle$ ground states are very long, while the encapsulated spin states in the fullerene also posses long $T_{1}$ times, 1 sec at $7 \mathrm{~K}[22]$, and probably several seconds for lower temperatures 21]. Thus the dominant source of decoherence in our model would be due to the hyperfine level broadening produced by the nuclei [14, 23]. This causes undesired dephasing in our scheme during the quantum tunneling of magnetization. We consider the SWAP time to be $2 \pi / \Omega+\mathrm{T}_{0}$ with $\mathrm{T}_{0}$ being the magnetization tunneling time. With current experimental techniques, $\Omega=20 \sim 30 \mathrm{MHz}$ is available [21], and $\mathrm{T}_{0}$ can be from nanosec to sec depending on the transverse magnetic field 24]. In the experiment of 25], it is shown that, in temperatures below $350 \mathrm{mK}$, and in the presence of $\mathrm{B}_{z}=42 \mathrm{mT}$ and a transverse B-field $200 \mathrm{mT}$, the line broadening is about $0.8 \mathrm{mT}$, corresponding to $22.4 \mathrm{MHz}$ in units of frequency. So if we assume $\Omega=30 \mathrm{MHz}$, to carry out our scheme, we require that $\mathrm{T}_{0}$ be shorter than 71 nanosec.

Another point we should mention is the initial state preparation of $\mathrm{Fe}_{8}$. Since the detected spin inside the fullerene in the readout stage should be well polarized, we can simply convert the spin information from $H_{1}$ to $H_{2}$ by performing $\mathrm{CNOT}_{12}$, instead of SWAP. This would 
simplify the readout scheme we mentioned above. To this end, however, we need to precisely prepare the initial states of $\mathrm{Fe}_{8}$ to be $|10\rangle$ or $-|10\rangle$. This too can be done by the quantum tunneling of magnetization. When we sweep the $\mathrm{B}_{z}$ field quickly, we can have an oscillation between $|10\rangle$ and $|-10\rangle$, whose frequency is heavily related to the tunnel splitting. By stopping the sweep field at an exactly chosen time, we can have a perfect initial state $|10\rangle$ or $|-10\rangle$ of $\mathrm{Fe}_{8}$.

In summary, we have proposed a potential method to efficiently detect the single spin state inside the fullerene by means of an auxiliary large spin nanomagnet. Since the spin states of the large-spin nanomagnet are measurable with current experimental techniques (e.g. Micro-
SQUID), we can reliably detect the qubit-encoded spin states inside the fullerene by swapping the detected state into the ground states of a nanomagnet.

\section{ACKNOWLEDGMENT}

The authors acknowledge thankfully the discussion with Wolfgang Wernsdorfer, Derek Mc Hugh and Graham Kells. MF is grateful for support from Chinese Academy of Sciences. The work is supported by EU Research Project QIPDDF-ROSES under contract number IST-2001-37150.
[1] See special issue, Fortschr. Phys. 48, (2000).

[2] B.E. Kane, Nature, 393, 133 (1998); B.E. Kane, N.S. MaAlpine, A.S. Dzurak, R.G. Clark, G.J. Milburn, H.B. Sun and H. Wiseman, Phys. Rev. B 61, 2961 (2000).

[3] H.-A. Engel and D. Loss, Phys. Rev. Lett. 86, 4648 (2001); P. Recher, E.V. Sukhorukov and D. Loss, Phys. Rev. Lett. 85, 1962 (2000).

[4] Y. Manassen, I. Mukhopadhyay and N.R. Rao, Phys. Rev. B 61, 16223 (2000); Y. Manassen, E. Terovanesyan, D. Shachal and S. Rivhter, Phys. Rev. B 48, 4887 (1993).

[5] G.P. Berman, G.D. Doolen, P.C. Hammel and V.I. Tsifrinovich, eprint, quant-ph/9909033 ibid, 0108025.

[6] D. Rugar, R. Budakian, H.J. Mamin, and B.W. Chul, Nature 430, 329 (2004).

[7] H. Park, J. Park, A.K.L. Lim, E.H. Anderson, A.P. Allvisatos and P.L. McEuen, Nature, 407, 57 (2000).

[8] M. Feng and J. Twamley, Phys. Rev. A 70, R030303 (2004).

[9] C.I. Pakes, P.W. Josephs-Franks, R.P. Reed, S.G. Corner and M.S. Colclough, IEEE Trans. Instr. and Meas. 50, 310 (2001).

[10] W. Harneit, Phys. Rev. A 65, 032322 (2002).

[11] D. Suter and K. Lim, Phys. Rev. A 65, 052309 (2002).

[12] J. Twamley, Phys. Rev. A 67, 052318 (2003).

[13] We can also employ the molecular nanomagnet $\mathrm{Mn}_{12}$ which has a large total spin $S=10$. But as shown in [14], even in the temperature below $60 \mathrm{mK}$, the excited states of $\mathrm{Mn}_{12}$ are still populated. This would affect our scheme. So we choose $\mathrm{Fe}_{8}$. But since $\mathrm{Mn}_{12}$ and $\mathrm{Fe}_{8}$ have many common characters, we will also cite some papers for $\mathrm{Mn}_{12}$ in our discussion.

[14] W. Wernsdorfer and R. Sessoli, Science, 284, 133 (1999).

[15] M.N. Leuenberger and D. Loss, Nature, 410, 789 (2001).

[16] C. Zener, Proc. R. Soc. London A 137, 696 (1932); A. Garg, Europhys. Lett. 22, 205 (1993).

[17] W. Wernsdorfer, N. Allaga-Alcalde, D.N. Hendrickson and G. Christou, Nature, 416, 406 (2002).

[18] Since there is no experimental data for that, this value is theoretically supposed due to the following fact: If a nanomagnet $\mathrm{Fe}_{8}$ with the same size as a fullerene $\mathrm{C}_{60}(<$ $0.6 \mathrm{~nm}$ ) is put next to a $\mathrm{C}_{60}$ by a spin-spin distance of 1 $\mathrm{nm}$, the dipolar coupling between them should be similar to that between two fullerenes [10] with the coupling strength $50 \mathrm{MHz}$. Considering the larger spin of the $\mathrm{Fe}_{8}$, we assume the dipolar coupling here is larger than in 10 by 7 times.

[19] W. Wernsdorfer, E.B. Orozoo, K. Hasselbach, A. Benoit, B. Barbara, N. Demoncy, A. Loiseau, D. Boivin, H. Pascard and D. Mailly, Phys. Rev. Lett. 78, 1791 (1997).

[20] A.L. Barra, D. Gatteschi and R. Sessoli, Phys. Rev. B 56, 8192 (1997); I. Mirebeau, M. Hennion, H. Casalta, H. Andres, H.U. Güdel, A.V. Irodova and A. Caneschi, Phys. Rev. Lett. 83, 628 (1999).

[21] C. Meyer, PhD thesis, Hahn-Meitner Institute Berlin, (2003).

[22] S. Knorr, A. Grupp, M. Mehring, M. Waiblinger and A. Weidinger, AIP Conference Proceedings, 544, 191 (2000).

[23] R. Sessoli, A. Caneschi, D. Gattesch, L. Sorace, A. Cornia and W. Wernsdorfer, J. Magn. Magn. Mater. 226-230, 1954 (2001).

[24] W. Wernsdorfer, private communication.

[25] W. Wernsdorfer, A. Caneschi, R. Sessoli, D. Gatteschi, A. Cornia, V. Villar, and C. Paulsen, Phys. Rev. Lett. 84, 2965 (2000). 
TABLE I: The eigenstates and the corresponding eigenenergies obtained from Eq. (3), where the degenerate transition frequencies between two nearest neighbor rows in the same column are listed in the last row. Due to the axial anisotropy term, there is no degenerate transition between two nearestneighbor columns. See text for the details.

\begin{tabular}{c|c|c|c|c}
\hline$\left|\frac{3}{2}, 10\right\rangle$ & $\left|\frac{3}{2}, 9\right\rangle$ & $\cdots \cdots$ & $\left|\frac{3}{2},-9\right\rangle$ & $\left|\frac{3}{2},-10\right\rangle$ \\
\hline$-11.5 \omega-100 D+15 J$ & $-10.5 \omega-81 D+13.5 J$ & $\cdots \cdots$ & $7.5 \omega-81 D-13.5 J$ & $8.5 \omega-100 D-15 J$ \\
\hline$\left|\frac{1}{2}, 10\right\rangle$ & $\left|\frac{1}{2}, 9\right\rangle$ & $\cdots \cdots$ & $\left|\frac{1}{2},-9\right\rangle$ & $\left|\frac{1}{2},-10\right\rangle$, \\
\hline$-10.5 \omega-100 D+5 J$ & $-9.5 \omega-81 D+4.5 J$ & $\cdots \cdots$ & $8.5 \omega-81 D-4.5 J$ & $9.5 \omega-100 D-5 J$ \\
\hline$\left|-\frac{1}{2}, 10\right\rangle$ & $\left|-\frac{1}{2}, 9\right\rangle$ & $\cdots \cdots$ & $\left|-\frac{1}{2},-9\right\rangle$ & $\left|-\frac{1}{2},-10\right\rangle$, \\
\hline$-9.5 \omega-100 D-5 J$ & $-8.5 \omega-81 D-4.5 J$ & $\cdots \cdots$ & $9.5 \omega-81 D+4.5 J$ & $10.5 \omega-100 D+5 J$ \\
\hline$\left|-\frac{3}{2}, 10\right\rangle$ & $\left|-\frac{3}{2}, 9\right\rangle$ & $\cdots \cdots$ & $\left|-\frac{3}{2},-9\right\rangle$ & $\left|-\frac{3}{2},-10\right\rangle$ \\
\hline$-8.5 \omega-100 D-15 J$ & $-7.5 \omega-81 D-13.5 J$ & $\cdots \cdots$ & $10.5 \omega-81 D+13.5 J$ & $11.5 \omega-100 D+15 J$ \\
\hline \hline$-\omega+10 J$ & $-\omega+9 J$ & $\cdots \cdots$ & $-\omega-9 J$ & $-\omega-10 J$ \\
\hline & \multicolumn{3}{|c}{}
\end{tabular}




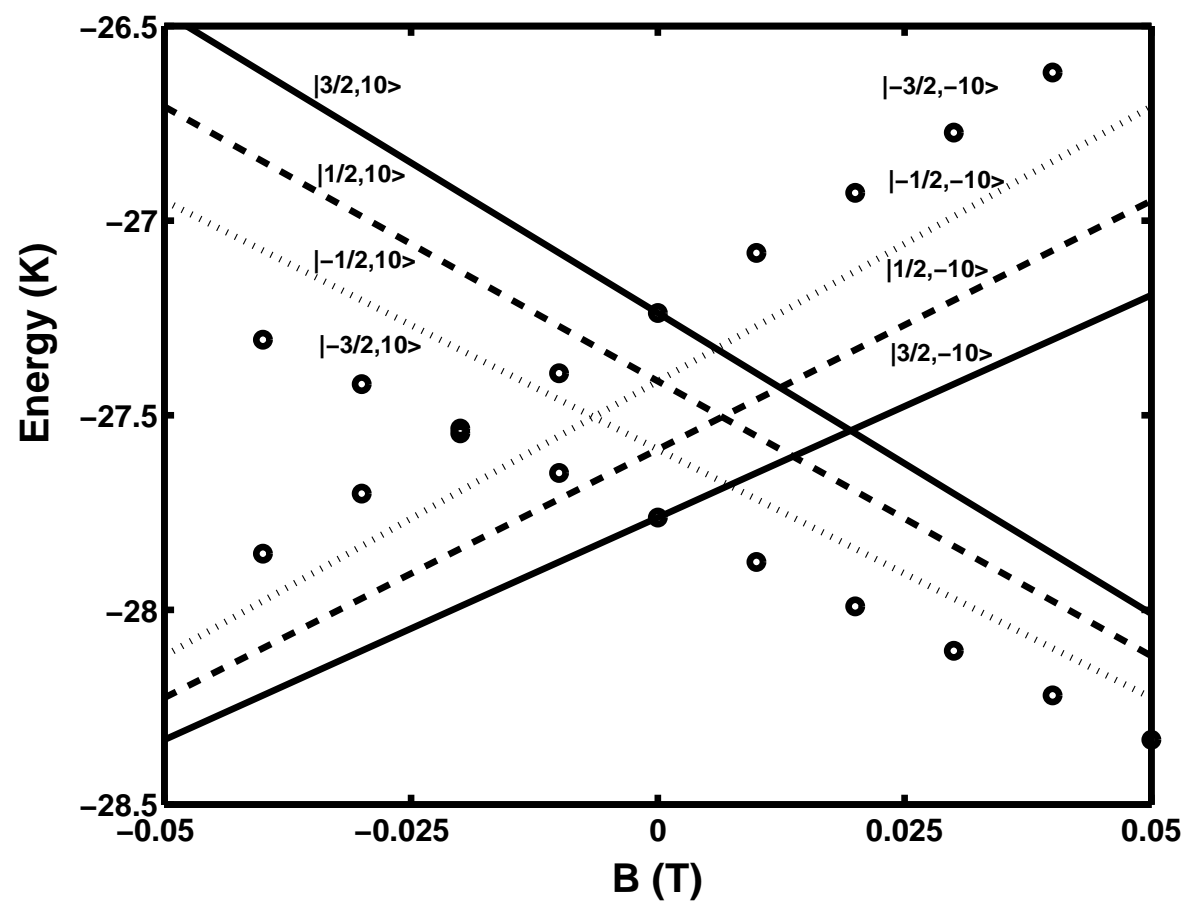

FIG. 1: Energy versus $B_{z}$ field plot for the low-lying states at very low temperature based on Eq. (3), where $D=0.275 \mathrm{~K}$ and $J=0.0175 K$. The quantum tunneling of magnetization happens at (avoided) crossing points of the same kinds of lines. The (avoided) crossing points of different kinds of lines corresponds to the magnetization tunneling with smaller probability due to second or higher order process. 